\title{
General polygamy inequality of multi-party quantum entanglement
}

\author{
Jeong San Kin* \\ Department of Mathematics, University of Suwon, Kyungki-do 445-743, Korea
}

(Dated: August 18, 2018)

\begin{abstract}
Using entanglement of assistance, we establish a general polygamy inequality of multi-party entanglement in arbitrary dimensional quantum systems. For multi-party closed quantum systems, we relate our result with the monogamy of entanglement to show that the entropy of entanglement is an universal entanglement measure that bounds both monogamy and polygamy of multi-party quantum entanglement.
\end{abstract}

PACS numbers: 03.67.Mn, 03.65.Ud

Entanglement has been recognized as a key resource in many quantum information processing tasks such as quantum teleportation [1] and dense coding [2]. Entanglement can be used to perform quantum key distribution where entangled-state analysis is used to prove the security of privacy amplification [3, 4] . Entanglement is also essential in certain models of quantum computing such as non-deterministic gate teleportation [5] and one-way quantum computation [6].

One distinct phenomenon of quantum entanglement from classical correlation is that it cannot be shared freely in multi-party systems. A simple example arises when a pair of parties in a multi-party quantum system share maximal entanglement, in which case they cannot have any entanglement nor classical correlations with other parties. This restricted shareability of entanglement in multi-party quantum systems is known as the Monogamy of Entanglement (MoE) 7]. MoE does not have any classical counterpart because all classical probability distributions can be shared among parties, and correlation between a pair of parties (whether they are perfectly correlated or not) does not restrict other parties' correlation. Thus MoE makes quantum physics fundamentally different form classical physics.

The first mathematical characterization of MoE was established by Coffman-Kundu-Wootters (CKW) for threequbit systems using tangle as the bipartite entanglement measure [8]. For a three-qubit state $\rho_{A B C}$ with twoqubit reduced density matrices $\operatorname{tr}_{C}|\psi\rangle_{A B C}\langle\psi|=\rho_{A B}$ and $\operatorname{tr}_{B}|\psi\rangle_{A B C}\langle\psi|=\rho_{A C}$,

$$
\tau\left(\rho_{A(B C)}\right) \geq \tau\left(\rho_{A B}\right)+\tau\left(\rho_{A C}\right)
$$

where $\tau\left(\rho_{A(B C)}\right)$ is the entanglement of $\rho_{A B C}$ with respect to the bipartition between $A$ and $B C$ measured by tangle, $\tau\left(\rho_{A B}\right)$ and $\tau\left(\rho_{A C}\right)$ are tangle of $\rho_{A B}$ and $\rho_{A C}$ respectively. Inequality (11) (also referred as CKW inequality) shows the mutually exclusive nature of multiparty quantum entanglement in a quantitative way; more entanglement shared between two qubits $(A$ and $B)$ necessarily implies less entanglement between the other two

*Electronic address: freddie1@suwon.ac.kr qubits $(A$ and $C)$. Later, CKW inequality was generalized for multi-qubit systems rather than just three qubits [9].

However, CKW inequality is know to fail in its generalization for higher-dimensional quantum systems due to the existence of quantum states violating Inequality (10) 10]. Moreover, this characterization of MoE in forms of an inequality is not generally true even in threequbit systems for other entanglement measures; one can easily find an example of three-qubit state that violates CKW inequality if we use Entanglement of Formation (EoF) [11] instead of tangle. Thus having a proper way of quantifying bipartite entanglement is crucial in the study of MoE.

Later, monogamy of multi-qubit entanglement and some cases of higher-dimensional quantum systems were characterized in terms of various entanglement measures [10, 12 14]. For general monogamy inequality of multi-part entanglement, it was recently shown that squashed entanglement [15] is a faithful entanglement measure (it vanishes only for separable states) [16], which also shows monogamy inequality of multi-party entanglement in arbitrary dimensional quantum systems [17].

Whereas MoE is about the restricted shareability of bipartite entanglement in multi-party quantum systems, the dual concept of bipartite entanglement namely Entanglement of Assistance (EoA) is known to have a dually monogamous (thus polygamous) property in multipartite quantum systems; for a three-qubit pure state $|\psi\rangle_{A B C}$, Polygamy of Entanglement (PoE) was characterized as a dual inequality 18, 19]

$$
\tau\left(|\psi\rangle_{A(B C)}\right) \leq \tau_{a}\left(\rho_{A B}\right)+\tau_{a}\left(\rho_{A C}\right),
$$

where $\tau_{a}\left(\rho_{A B}\right)$ and $\tau_{a}\left(\rho_{A C}\right)$ are the tangle of assistance [19] of $\rho_{A B}$ and $\rho_{A C}$ respectively.

For $\mathrm{MoE}$ characterized by CKW inequality, the bipartite entanglement between $A$ and $B C$ measured by tangle is an upper bound for the sum of two-qubit entanglement between $A$ and each of $B$ and $C$. Interestingly, the same quantity also plays as a lower bound for the sum of two-qubit entanglement of assistance in the polygamy inequality. Later $\mathrm{PoE}$ was generalized into multi-qubit systems [13, 19] and three-party pure states of arbitrary dimension [20]. Recently, a tight upper bound on polygamy 
inequality was also proposed in an arbitrary-dimensional multi-party quantum systems [21]. However, a general polygamy inequality of multi-party, higher-dimensional quantum systems was an open question.

The study of quantum entanglement in higherdimensional quantum systems is important for not only theoretical sense but practical reasons as well; MoE can restrict the possible correlation between authorized users and the eavesdropper, which tightens security bounds in quantum cryptography (QC). Furthermore, to optimize the efficiency of entanglement usage as a resource in $\mathrm{QC}$, higher-dimensional quantum systems rather than qubits are preferred in some physical systems for stronger security in quantum key distribution (QKD) 22]. However, generalization from qubits to qudits is not straightforward for example, in the complexity of a no-go theorem for universal transversal gates in quantum error correlation [23].

Here, we provide a polygamy inequality of entanglement that holds for multi-party quantum systems of arbitrary high dimensions. For multi-party closed quantum systems, we relate our result with the monogamy inequality [17], and show that the entropy of entanglement serves as both upper and lower bounds for monogamy and polygamy of multi-party quantum entanglement. Thus the entropy of entanglement is an universal entanglement measure that bounds both MoE and PoE.

Let us recall the definition of EoF and EoA of bipartite quantum systems. For a bipartite pure state $|\psi\rangle_{A B}$, its entropy of entanglement is

$$
E\left(|\psi\rangle_{A B}\right):=S\left(\rho_{A}\right)
$$

where $\rho_{A}=\operatorname{tr}_{B}|\psi\rangle_{A B}\langle\psi|$ and $S(\rho)=-\operatorname{tr} \rho \log \rho$ is von Neumann entropy. For a mixed state $\rho_{A B}$, its EoF is defined as the minimum average entanglement

$$
E_{f}\left(\rho_{A B}\right)=\min \sum_{i} p_{i} E\left(|\psi\rangle_{A B}\right),
$$

where the minimization is taken over all possible purestate decompositions of $\rho_{A B}=\sum_{i} p_{i}\left|\psi^{i}\right\rangle_{A B}\left\langle\psi^{i}\right|$. This procedure of minimizing over all pure-state decompositions to determine mixed-state entanglement is known as the convex-roof extension. From the convex-roof nature inherent in the definition, EoF of $\rho_{A B}$ is considered as the minimum amount of entanglement needed to prepare $\rho_{A B}$, hence the terminology formation.

As a dual quantity to EoF, EoA of $\rho_{A B}$ is defined as the maximum average entanglement

$$
E_{a}\left(\rho_{A B}\right)=\max \sum_{i} p_{i} E\left(|\psi\rangle_{A B}\right),
$$

over all possible pure-state decompositions of $\rho_{A B}$ [24]. If we consider $\rho_{A B}$ together with a purification $|\psi\rangle_{A B C}$ such that $\rho_{A B}=\operatorname{tr}|\psi\rangle_{\mathrm{ABC}}\langle\psi|$, the party $C$ having the purification of $\rho_{A B}$ can help increasing the entanglement of $\rho_{A B}$ by performing measurements on its own system
$C$ and communicating the measurement results to $A$ and $B$.

Furthermore, the one-to-one correspondence between rank-one measurements of $C$ and pure state ensembles of $\rho_{A B}$ makes this maximum possible average entanglement between $A$ and $B$ with the assistance of $C$ as an intrinsic definition; the maximum average entanglement over all possible pure-state decompositions of $\rho_{A B}$, which is the definition of EoA in Eq. (5). Thus, not only the mathematical duality between EoF and EoA (one takes the minimum whereas the other one takes the maximum), they also have physical interpretations that are dual to each other; one is the concept of formation and the other is the possible achievable entanglement with assistance of the environment.

Before we discuss polygamy relation of multi-party entanglement in terms of EoA, let us first consider a tradeoff relation between EoA and one-way unlocalizable entanglement (UE) in three-party quantum systems [20]; for a three-party pure state $|\psi\rangle_{A B C}$ with reduced density matrices $\rho_{A B}$ and $\rho_{A C}$, we have

$$
S\left(\rho_{A}\right)=E_{u}^{\leftarrow}\left(\rho_{A B}\right)+E_{a}\left(\rho_{A C}\right),
$$

where $E_{u}^{\leftarrow}\left(\rho_{A B}\right)$ is UE of $\rho_{A B}$

$$
E_{u}^{\leftarrow}\left(\rho_{A B}\right):=\min _{\left\{M_{x}\right\}}\left[S\left(\rho_{A}\right)-\sum_{x} p_{x} S\left(\rho_{A}^{x}\right)\right],
$$

with the minimum being taken over all possible rank-1 measurements $\left\{M_{x}\right\}$ applied on system $B$.

Eq. (6) implies that the entropy of entanglement of $|\psi\rangle_{A B C}$ with respect to the bipartition between $A$ and $B C$ consists of two distinct parts: one is the robust entanglement that can be localized onto $A C$ after the local measurement of $B$ (denoted by $E_{a}\left(\rho_{A C}\right)$ ), and the other part is too sensitive to be localized onto $A C$ (denoted by $\left.E_{u}^{\leftarrow}\left(\rho_{A B}\right)\right)$.

UE is known to be subadditive under tensor product of quantum states, and bounded below by the coherent information [20]. Furthermore, form the quantitative relation between UE and mutual information

$$
E_{u}^{\leftarrow}\left(\rho_{A B}\right) \leq \frac{I\left(\rho_{A B}\right)}{2},
$$

Eq. (6) was shown to imply a trade-off relation of localizable entanglement measured by EoA in three-party quantum systems; for any tripartite pure state $|\psi\rangle_{A B C}$,

$$
S\left(\rho_{A}\right) \leq E_{a}\left(\rho_{A B}\right)+E_{a}\left(\rho_{A C}\right) .
$$

Thus, for a tripartite pure state of arbitrary dimension, there always exists a polygamy relation of localizable entanglement that can be quantified by the entropy of entanglement and EoA, which is illustrated in Figure 1.

Now we generalize Inequality (9) for arbitrary mixed states of multi-party quantum systems rather than just three parties. We first show that Inequality (9) is true for tripartite mixed states, and use the result to establish a 


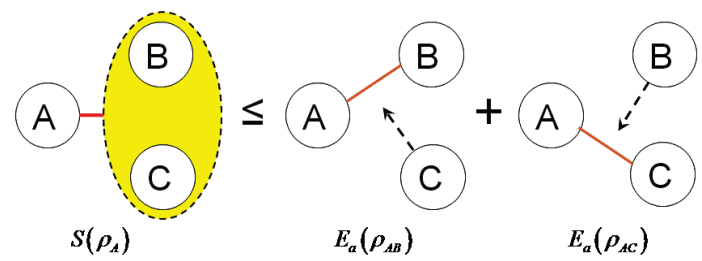

Fig. 1: The entanglement between $A$ and $B C$ of a three-party pure state $|\psi\rangle_{A(B C)}$ measured by $S\left(\rho_{A}\right)$ is always bounded by the sum of localizable entanglement between $A$ and $B$ measured by $E_{a}\left(\rho_{A B}\right)$, and between $A$ and $C$ measured by $E_{a}\left(\rho_{A C}\right)$.

general polygamy inequality of EoA in arbitrary dimensional multi-party quantum systems. For a three-party mixed state $\rho_{A B C}$, let $\rho_{A(B C)}=\sum_{j} p_{j}\left|\psi_{j}\right\rangle_{A(B C)}\left\langle\psi^{j}\right|$ be an optimal decomposition for EoA with respect to the bipartition between $A$ and $B C$,

$$
E_{a}\left(\rho_{A(B C)}\right)=\sum_{j} p_{j} E\left(\left|\psi^{j}\right\rangle_{A(B C)}\right)
$$

where $E\left(\left|\psi^{j}\right\rangle_{A(B C)}\right)=S\left(\rho_{A}^{j}\right)$ is the pure-state entanglement of $\left|\psi^{j}\right\rangle_{A(B C)}$ for each $j$ with $\rho_{A}^{j}=$ $\operatorname{tr}_{B C}\left|\psi^{j}\right\rangle_{A B C}\left\langle\psi^{j}\right|$.

For each $j,\left|\psi^{j}\right\rangle_{A B C}$ is a tripartite pure state, therefore Inequality (9) leads us to

$$
\begin{aligned}
E\left(\left|\psi^{j}\right\rangle_{A(B C)}\right) & =S\left(\rho_{A}^{j}\right) \\
& \leq E_{a}\left(\rho_{A B}^{j}\right)+E_{a}\left(\rho_{A C}^{j}\right)
\end{aligned}
$$

with $\rho_{A B}^{j}=\operatorname{tr}_{C}\left|\psi^{j}\right\rangle_{A B C}\left\langle\psi^{j}\right|$ and $\rho_{A C}^{j}=$ $\operatorname{tr}_{B}\left|\psi^{j}\right\rangle_{A B C}\left\langle\psi^{j}\right|$. The linearity of partial trace implies

$$
\sum_{j} p_{j} \rho_{A B}^{j}=\rho_{A B}, \quad \sum_{j} p_{j} \rho_{A C}^{j}=\rho_{A C},
$$

and together with the definition of EoA, we have

$$
\begin{aligned}
& \sum_{j} p_{j} E_{a}\left(\rho_{A B}^{j}\right) \leq E_{a}\left(\rho_{A B}\right), \\
& \sum_{j} p_{j} E_{a}\left(\rho_{A C}^{j}\right) \leq E_{a}\left(\rho_{A C}\right) .
\end{aligned}
$$

From the inequalities (11), (13) and Eq. (10), we thus have

$$
\begin{aligned}
E_{a}\left(\rho_{A(B C)}\right) & =\sum_{j} p_{j} E\left(\left|\psi^{j}\right\rangle_{A(B C)}\right) \\
& \leq \sum_{j} p_{j} E_{a}\left(\rho_{A B}^{j}\right)+\sum_{j} p_{j} E_{a}\left(\rho_{A C}^{j}\right) \\
& \leq E_{a}\left(\rho_{A B}\right)+E_{a}\left(\rho_{A C}\right) .
\end{aligned}
$$

In other words, Inequality (9) can be generalized for tripartite mixed states in terms of EoA.

Now let us consider a multi-party quantum state $\rho_{A_{1} A_{2} \cdots A_{n}}$ rather than just three parties. By letting $A_{1}=A, A_{2}=B$ and $A_{3} \cdots A_{n}=C$, we can consider $\rho_{A_{1} A_{2} \cdots A_{n}}$ as a tripartite quantum state, and Inequality (14) leads us to

$$
E_{a}\left(\rho_{A_{1}\left(A_{2} \cdots A_{n}\right)}\right) \leq E_{a}\left(\rho_{A_{1} A_{2}}\right)+E_{a}\left(\rho_{A_{1}\left(A_{3} \cdots A_{n}\right)}\right),
$$

where $\rho_{A_{1} A_{2}}=\operatorname{tr}_{A_{3} \cdots A_{n}} \rho_{A_{1} A_{2} \cdots A_{n}}, \quad \rho_{A_{1} A_{3} \cdots A_{n}}=$ $\operatorname{tr}_{A_{2}} \rho_{A_{1} A_{2} \cdots A_{n}}$, and $\left.E_{a}\left(\rho_{A_{1}\left(A_{3} \cdots A_{n}\right.}\right)\right)$ is EoA of $\rho_{A_{1} A_{3} \cdots A_{n}}$ with respect to the bipartition between $A_{1}$ and $A_{3} \cdots A_{n}$. Because $\rho_{A_{1} A_{3} \cdots A_{n}}$ in Inequality (15) is a $(n-1)$-party quantum state, we can apply Inequality (14) to obtain $E_{a}\left(\rho_{A_{1}\left(A_{3} \cdots A_{n}\right)}\right) \leq E_{a}\left(\rho_{A_{1} A_{3}}\right)+E_{a}\left(\rho_{A_{1}\left(A_{4} \cdots A_{n}\right)}\right)$. Thus, by iterating Inequality (14) on the last term of Inequality (15), we obtain the following polygamy inequality of multi-party entanglement

$$
E_{a}\left(\rho_{A_{1}\left(A_{2} \cdots A_{n}\right)}\right) \leq E_{a}\left(\rho_{A_{1} A_{2}}\right)+\cdots+E_{a}\left(\rho_{A_{1} A_{n}}\right) .
$$

In contrast to monogamy inequality, which provides an upper bound on the shareability of bipartite entanglement in multi-party quantum systems, polygamy inequality in (16) provides a lower bound of how much entanglement can be created on bipartite subsystems with assistance of the other parties.

Here we further note that this upper and lower bounds of entanglement distribution in multi-party quantum systems can be determined by a single quantity, the entropy of entanglement for closed systems. The result about three-party monogamy inequality in terms of squashed entanglement (SE) in [17] can easily be generalized into an arbitrary multi-party quantum system; for a $n$-party state $\rho_{A_{1} A_{2} \cdots A_{n}}$,

$$
E_{s q}\left(\rho_{A_{1}\left(A_{2} \cdots A_{n}\right)}\right) \geq E_{s q}\left(\rho_{A_{1} A_{2}}\right)+\cdots+E_{s q}\left(\rho_{A_{1} A_{2}}\right),
$$

where $E_{s q}\left(\rho_{A B}\right)$ is $\mathrm{SE}$ of $\rho_{A B}$ defined as

$E_{s q}\left(\rho_{\mathrm{AB}}\right):=\frac{\inf \left\{S\left(\rho_{A E}\right)+S\left(\rho_{B E}\right)-S\left(\rho_{A B E}\right)-S\left(\rho_{E}\right)\right\}}{2}$

with the infimum taken over all possible extension $\rho_{A B E}$ of $\rho_{A B}$ such that $\operatorname{tr}_{E} \rho_{A B E}=\rho_{A B}$. The quantity inside the parenthesis of Eq. (18) is quantum conditional mutual information of $\rho_{A B E}$ denoted by $I(A ; B \mid E)$.

For a bipartite pure state $|\psi\rangle_{A B}$, any possible extension $\rho_{A B C}$ such that $\operatorname{tr}_{C} \rho_{A B C}=|\psi\rangle_{A B}\langle\psi|$ must be a product state $|\psi\rangle_{A B}\langle\psi| \otimes \rho_{C}$ for some $\rho_{C}$ of subsystem $C$. From this fact, it is also straightforward to verify that SE in Eq. (18) coincides with $S\left(\rho_{A}\right)$ for any pure state $|\psi\rangle_{A B}$ with reduced density matrix $\rho_{\mathrm{A}}$. In other words, for a multi-party closed quantum system described by a pure state $|\psi\rangle_{A_{1} A_{2} \cdots A_{n}}$, the monogamy inequality in 
terms of SE in (17) becomes

$$
S\left(\rho_{A_{1}}\right) \geq E_{s q}\left(\rho_{A_{1} A_{2}}\right)+\cdots+E_{s q}\left(\rho_{A_{1} A_{2}}\right),
$$

where $S\left(\rho_{A_{1}}\right)=E\left(|\psi\rangle_{A_{1}\left(A_{2} \cdots A_{n}\right)}\right)$ is the entropy of entanglement of the pure state $|\psi\rangle_{A_{1} A_{2} \cdots A_{n}}$ with respect to the bipartition between $A_{1}$ and the other parties. Furthermore, from the definition of EoA in Eq. (5), we also note that the left-hand side of polygamy inequality in (16) becomes the entropy of entanglement,

$$
S\left(\rho_{A_{1}}\right) \leq E_{a}\left(\rho_{A_{1} A_{2}}\right)+\cdots+E_{a}\left(\rho_{A_{1} A_{n}}\right),
$$

for this closed quantum system described by $|\psi\rangle_{A_{1} A_{2} \cdots A_{n}}$. Thus the entropy of entanglement quantifying bipartite pure-state entanglement is an universal entanglement measure that provides bounds for both monogamy and polygamy of multi-party quantum entanglement.

To summarize, we have shown the polygamous nature of bipartite entanglement distribution in multipar- tite quantum systems; using EoA, we establish a general polygamy inequality of multi-party entanglement in arbitrary high-dimensional quantum systems rather than just qubits. For multi-party closed quantum systems, we have related our polygamy inequality with the monogamy inequality in terms of SE, and clarified that the entropy of entanglement serves as both upper and lower bounds for MoE and PoE.

Our result completely characterizes the polygamous nature of entanglement distribution in multi-party quantum systems of arbitrary high dimension. Noting the importance of the study on high-dimensional multipartite entanglement, our result can provide a rich reference for future work on the study of multipartite entanglement.

\section{Acknowledgments}

This work was supported by Emerging Technology R\&D Center of SK Telecom.
[1] C. H. Bennett, G. Brassard, C. Crepeau, R. Jozsa, A. Peres and W. K. Wootters, Phys. Rev. Lett. 70, 1895 (1993).

[2] C. H. Bennett and S. J. Wiesner, Phys. Rev. Lett. 69, 2881 (1992).

[3] C. Bennett and G. Brassard, in Proceedings of IEEE International Conference on Computers, Systems, and Signal Processing (IEEE Press, New York, Bangalore, India, 1984), p. $175-179$.

[4] A. Ekert, Phys. Rev. Lett. 67, p. 661-663 (1991).

[5] M. A. Nielsen and I. L. Chuang, Phys. Rev. Lett. 79, 321 (1997); M. Hillery, M. Ziman, and V. Bužek, Phys. Rev. A 73, 022345 (2006); M. Mičuda, M. Ježek, M. Dušek and J. Fiurášek, Phys. Rev. A 78, 062311 (2008).

[6] R. Raussendorf and H.J. Briegel, Phys. Rev. Lett. 86, 5188 (2001).

[7] B. M. Terhal, IBM J. Research and Development 48, 71 (2004).

[8] V. Coffman, J. Kundu and W. K. Wootters, Phys. Rev. A 61, 052306 (2000).

[9] T. Osborne and F. Verstraete, Phys. Rev. Lett. 96, 220503 (2006).

[10] J. S. Kim, A. Das and B. C. Sanders, Phys. Rev. A 79, 012329 (2009).

[11] C. H. Bennett, D. P. DiVincenzo, J. A. Smolin and W. K. Wootters, Physical Review A 54, 3824 (1996).
[12] J. S. Kim and B. C. Sanders, J. Phys. A: Math. and Theor. 43, 445305 (2010).

[13] J. S. Kim, Phys. Rev. A. 81, 062328 (2010).

[14] J. S. Kim and B. C. Sanders, J. Phys. A: Math. and Theor. 44, 295303 (2011).

[15] M. Christandl and A. Winter, J. Math. Phys. 45, p. 829 840 (2004).

[16] F. G. S. L. Brandao, M. Christandl and J. Yard, Commun. Math. Phys. 306, 805 (2011).

[17] M. Koashi and A. Winter, Phys. Rev. A 69, 022309 (2004).

[18] G. Gour, D. Meyer and B. C. Sanders, Phys. Rev. A 72, 042329 (2005).

[19] G. Gour, S. Bandyopadhay and B. C. Sanders, J. Math. Phys. 48, 012108 (2007).

[20] F. Buscemi, G. Gour and J. S. Kim, Phys. Rev. A 80, 012324 (2009).

[21] J. S. Kim, Phys. Rev. A 80, 022302 (2009).

[22] S. Groblacher, T. Jennewein, A. Vaziri, G. Weihs and A. Zeilinger, New J. Phys. 8, 75 (2006).

[23] X. Chen, H. Chung, A. W. Cross, B. Zeng and I. L. Chuang, Phys. Rev. A 78, 012353 (2008).

[24] T. Laustsen, F. Verstraete and S.J. van Enk, Quantum Inf. Comput. 3, 64 (2003). 